\title{
Influence of the ionic character of a drug on its release rate from hydrogels based on 2-hydroxyethylmethacrylate and acrylamide synthesized by photopolymerization
}

\author{
M. L. Gómez ${ }^{1}$, R. J. J. Williams ${ }^{2}$, H. A. Montejano ${ }^{1}$, C. M. Previtali ${ }^{1}$ \\ ${ }^{1}$ Departamento de Química, Universidad Nacional de Río Cuarto y CONICET, Campus Universitario, 5800 Río Cuarto, \\ Argentina \\ ${ }^{2}$ Institute of Materials Science and Technology (INTEMA), University of Mar del Plata and National Research Council \\ (CONICET), J. B. Justo 4302, 7600 Mar del Plata, Argentina
}

Received 11 July 2011; accepted in revised form 24 September 2011

\begin{abstract}
The influence of the ionic character of a specific drug on its release rate from a hydrogel based on 2-hydroxyethylmethacrylate (HEMA) and acrylamide (AAm) is analyzed. The hydrogel was synthesized by photopolymerization employing visible light, safranine $\mathrm{O}$ (Saf), as sensitizer, and a silsesquioxane functionalized with amine and methacrylate groups (SFMA), as co-initiator and crosslinker. Safranine O (Saf) was employed as a model of a cationic drug and the anionic form of resorufin (Rf) as a model of an anionic drug. Saf exhibited a larger affinity with functional groups of the hydrogel than that of Rf. This produced a lower loading and a faster release rate of Rf with respect to Saf. Besides, the release rate of Rf followed a Fickian behavior, while that of Saf exhibited a non-Fickian behavior. By hydrolyzing the hydrogel at $\mathrm{pH}=13$, amide groups supplied by AAm were irreversibly converted into carboxylic acid groups. Higher loadings and slower release rates of Saf from the hydrolyzed hydrogels were observed, making them particularly suitable for the slow drug-delivery of cationic drugs.
\end{abstract}

Keywords: polymer gels, hydrogels, drug release, hydroxyethylmethacrylate, acrylamide

\section{Introduction}

Hydrogels are crosslinked hydrophilic polymers that constitute an important class of materials in biotechnology and medicine because of their excellent biocompatibility [1-2]. Over the past three decades, a variety of hydrogels differing in structure, composition, and properties were developed [3-4]. Hydrogels exhibiting a swelling response to environmental changes such as temperature, $\mathrm{pH}$, electric field, UV or visible light-radiation, solvent composition, salt concentration and type of surfactants are attracting increasing interest in various applications such as drug delivery systems, separation operations in biotechnology, processing of agricultural products, conductive or superabsorbent composites, and sensors and actuators [1, 5-7]. HEMA (2-hydroxyethylmethacrylate) was the first monomer used to synthesize hydrogels for biomedical applications [8]. Its water swelling properties are improved by co-polymerization with more hydrophilic monomers [7, 9-10].

Hydrogels may be synthesized via various polymerization techniques, such as thermal polymerization [11], oxidation-reduction (redox) polymerization [12] and photopolymerization [13-14]. Photopolymerization has several advantages over conventional polymerization techniques. These include spatial and temporal control over polymerization

\footnotetext{
${ }^{*}$ Corresponding author, e-mail: mlgomez@exa.unrc.edu.ar
} (c) BME-PT 
and fast curing rate at room or physiological temperatures. One major advantage of photopolymerization is that hydrogels can be synthesized in the presence of an active principle, facilitating the incorporation of drugs during the synthesis of the hydrogels. Photopolymerization has been employed to obtain hydrogels for drug delivery applications using a variety of mono functional monomers such as HEMA [7-12, 15], and crosslinkers such as poly (ethylene glycol) dimethacrylate [16], and poly(ethylene glycol) diacrylate [17]. The co-polymerization of HEMA, acrylamide (AAm) and a suitable crosslinker provides a unique combination of properties of the resulting hydrogels, making them capable of swelling especially at high $\mathrm{pH}$ values [18-19].

Water-soluble photoinitiator systems for vinyl polymerizations, above all those active in the visible region of the spectrum, have gained increasing interest in recent years [20]. Among them, the most commonly employed photoinitiators are those generating radicals by a bimolecular process comprising an excited state of a synthetic dye or natural pigment, and a co-initiator that behaves as electron donor. Particularly, safranine O, an azine dye, was extensively studied for its use as sensitizer of photopolymerization in organic and aqueous media employing visible light as energy source and different amines as co-initiators [21-24].

In a previous study we reported the synthesis of a silsesquioxane functionalized with methacrylate and amine groups (SFMA) that was employed as crosslinker/co-initiator to obtain poly(HEMA-coAAm) hydrogels by visible-light photopolymerization, employing safranine $\mathrm{O}$ (Saf) as sensitizer [19]. The swelling behavior of the poly(HEMA-coAAm) hydrogels at different $\mathrm{pHs}$ was also reported [19]. The present study focuses on the way in which the release rate of a specific compound retained inside the poly(HEMA-co-AAm) hydrogel is affected by the ionic character of the active species, using dyes as model drugs. Two dyes were used: safranine-O (Saf) as an axample of a cationic active group and resorufin (Rf) as an example of an anionic active group. While Saf is a strong base, resorufin is an acid with a $p K a=7.9$. A significant fraction of Saf persists in its cationic form even at high values of $\mathrm{pH}$. This is not the case for $\mathrm{Rf}$, where the fraction of the conjugated base (anion) is very low at low values of $\mathrm{pH}$. Therefore, experimental results with $\mathrm{Rf}$ were obtained at $\mathrm{pH}=7$.

The influence of $\mathrm{pH}$ and temperature on the release rate of dyes and drugs from specific hydrogels (including those based on HEMA) has been reported in a large number of papers [e.g., 7, 15, 25-40]. However, to the best of our knowledge there are no comparative studies of the release rate of salts with large ions and small counterions from a specific hydrogel under defined $\mathrm{pH}$ and temperature conditions. This is an important concept because several drugs are used as salts. The following are examples of release rate studies of cationic drugs: pralidoxime chloride [28], salbutamol sulfate [30], gentamicin sulfate [25], and anionic drugs: sodium sulfacetamide [25]. We employed safranine (Saf) as a model of a cationic drug and the anionic form of resorufin (Rf) as a model of an anionic drug.

The election of poly(HEMA-co-AAm) hydrogels for this study was based on two facts: a) this is a classic system for drug delivery studies, b) they can be hydrolyzed at $\mathrm{pH}=13$ to irreversibly transform their amide functionalities into carboxylate groups [19], a possibility that can be used to compare the behavior of poly(HEMA-co-AAm) and poly(HEMAco-AA) hydrogels of the same composition.

\section{Experimental \\ 2.1. Materials}

The silsesquioxane functionalized with methacrylate and amine groups (SFMA) was synthesized as described in a previous paper [19]. Acrylamide (AAm - Code: A8887 - Assay $\geq 99 \%$ ) and 2-hydroxyethylmethacrylate (HEMA - Code: 128635 - Assay: 97\%) were provided by Aldrich, Steinheim, Germany and used as received. Safranine O (Saf - Code: $\mathrm{S} 2255)$ and the anionic form of resorufin $(\mathrm{Rf}$ - Code: R3257) were purchased from Aldrich, Steinheim, Germany and employed without further purification. Water was purified through a Millipore MilliQ system. Buffer solutions were provided by Laboratorios Oliveri, Buenos Aires, Argentina and used as received. The structures of a representative molecule of SFMA, monomers and dyes are presented in Figure 1.

\subsection{Synthesis of hydrogels}

Hydrogels were synthesized employing the following proportions of monomers by weight: HEMA 

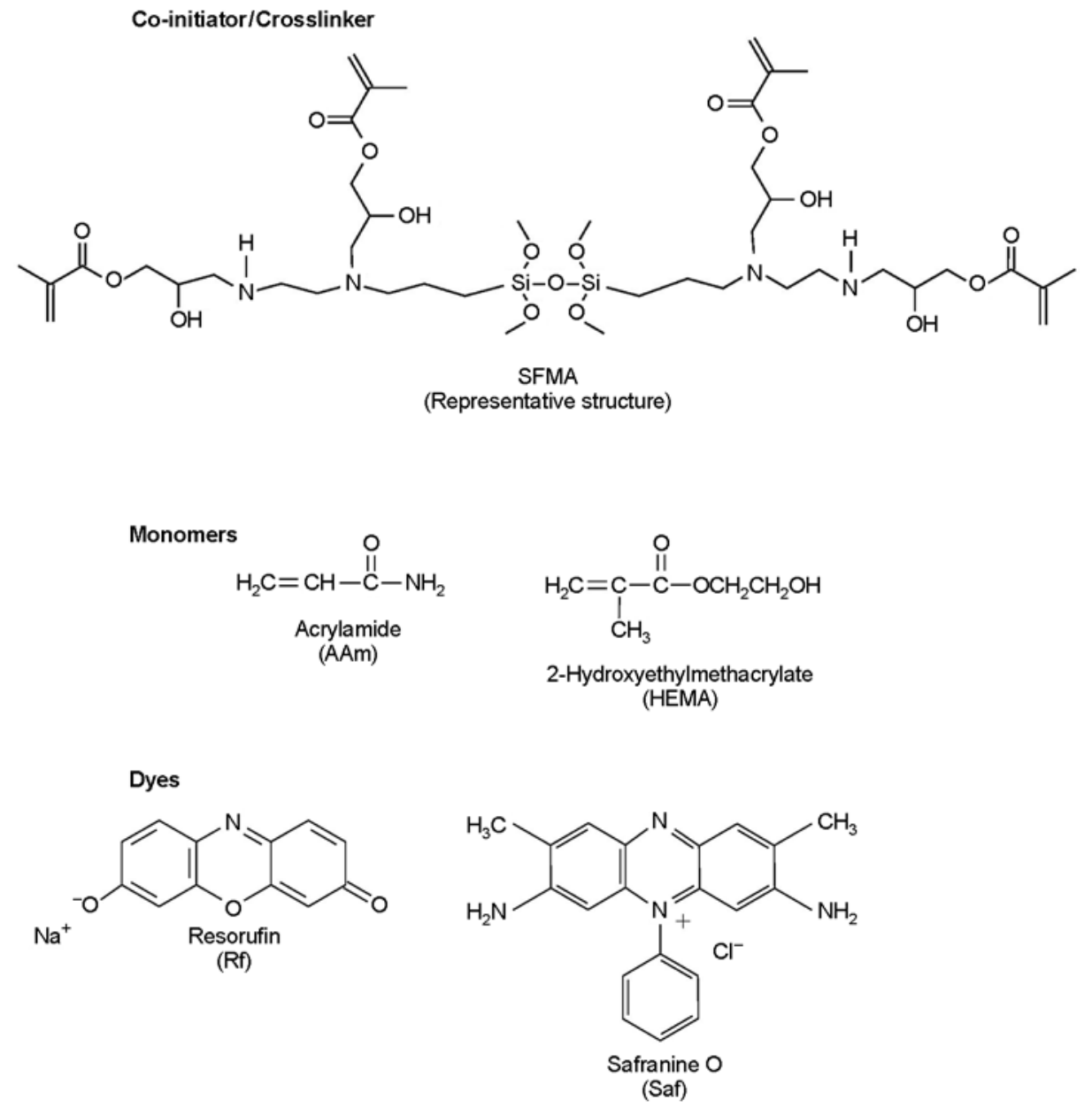

Figure 1. Structures of SFMA, monomers and model drugs

90\%, AAm 9\% and photoinitiator/crosslinker 1\%. The photoinitiator/crosslinker was composed of Saf/SFMA in similar proportions as in our previous study [19]. Typically, $2 \mathrm{~mL}$ of a deaerated aqueous solution ( 50 by volume) of this formulation was irradiated for $2 \mathrm{~h}$ in a homemade merry-go-round photochemical reactor supplied with eight green LEDs $\left(\lambda_{\max }=530 \mathrm{~nm}\right)$.

\subsection{Swelling}

Uniform disks, $10.5 \mathrm{~mm}$ diameter and $2.5 \mathrm{~mm}$ thickness, were cut from the hydrogels and immersed in Milli-Q water to remove unreacted monomers, Saf and SFMA; water was daily replaced for one week. After this period hydrogels were washed with Milli-Q water and dried in a vacuum oven at $37^{\circ} \mathrm{C}$ for $48 \mathrm{~h}$. Dried disks were weighed $\left(W_{\mathrm{pol}}\right)$ and immersed in commercial buffer solutions at room temperature. At specified times samples were removed from the solutions, blotted with filter paper to eliminate excess of solution and weighed $\left(W_{\mathrm{t}}\right)$. Three samples were used per point of the swelling curve. The degree of swelling $\left(S_{\mathrm{w}}\right)$ was calculated accoding to Equation (1):

$S_{\mathrm{w}}=\frac{W_{\mathrm{t}}-W_{\mathrm{pol}}}{W_{\mathrm{pol}}}=\frac{W_{\mathrm{water}}}{W_{\mathrm{pol}}}$

To visualize the pore structure of the hydrogels in the swollen state, a JEOL JSM-6460 LV SEM, from Jeol Technics Ltd., Tokyo, Japan was employed. Samples were swollen to equilibrium in buffer solutions, blotted with filter paper, frozen at $-18^{\circ} \mathrm{C}$ and freeze-dried for $24 \mathrm{~h}$ in a Virtis Benchtop SLC, SP Industries, New York, USA. Freeze-dried samples were loaded on the surface of an aluminum SEM specimen holder and sputter coated with Au-Pd for $35 \mathrm{~s}$ before observation. A working distance about 20-25 mm, an accelerating voltage of $15 \mathrm{kV}$, and a chamber pressure of $10^{-9}$ Torr were found to be suitable for obtaining high-resolution images. 


\subsection{Loading and release of dyes}

Disks of dried hydrogels were immersed for $24 \mathrm{~h}$ in aqueous solutions of the dyes with a concentration giving a maximum value of absorbance equal to 1.2 , measured with an optical path of $1 \mathrm{~cm}$. Disks were removed from the solution and dried under vacuum at $37^{\circ} \mathrm{C}$ for $48 \mathrm{~h}$. The amount of dye that was loaded was determined from the residual absorbance of the solution employing a calibration curve following Lambert-Beer's law. UV-vis spectra were recorded with an Agilent 8453 diode array spectrophotometer, Waldbronn, Germany. Samples were placed in a $1 \mathrm{~cm} \times 1 \mathrm{~cm} \times 3 \mathrm{~cm}$ quartz cell and spectra recorded at room temperature.

Release experiments were carried out by transferring the dried dye-loaded disks into $10 \mathrm{~mL}$ buffer solutions of different $\mathrm{pHs}$ at room temperature. At specified time intervals, $3 \mathrm{~mL}$ aliquots were removed from every solution (three aliquots of different solutions for any single point of the release curve), and their absorbance determined by ultraviolet-visible (UV-vis) spectroscopy at the maximum absorption wavelength of each dye. After measuring the absorbance, aliquots were returned to the original solutions to keep volume constant. Calibration curves were used to transform absorbance determinations into concentrations.
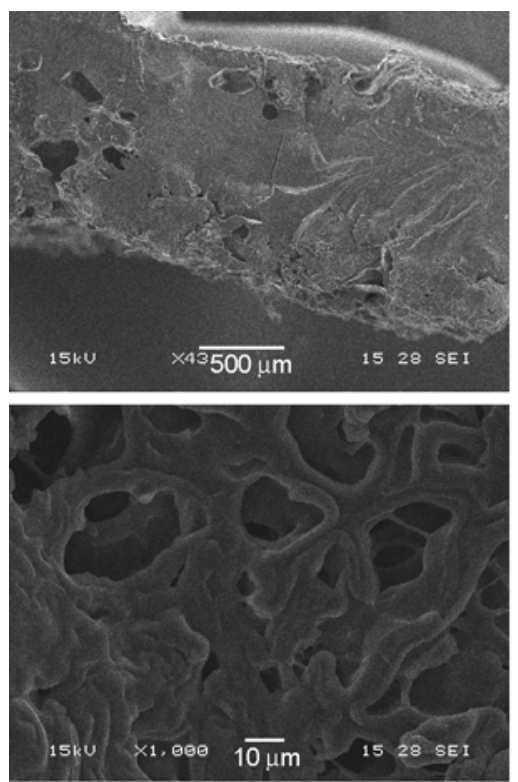

A)
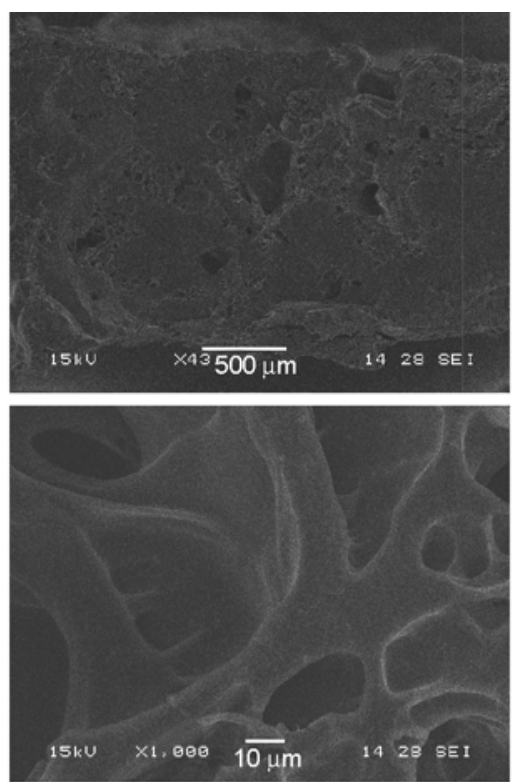

B)

\section{Results and discussion}

\subsection{Swelling behavior of hydrogels}

The swelling of the hydrogel in water at different $\mathrm{pH}$ values can be related to its behavior as a drugdelivery material. Preliminary swelling results of the synthesized hydrogels were reported in our previous study [19]. A significant increase in the swelling capacity was observed at $\mathrm{pH} 13$, explained by the almost complete conversion of acrylamide into carboxylate groups [19]. The repulsion of the anionic groups fixed in the gel structure and the increase in hydrophilicity produced by the presence of ionic species inside the gel led to an increase in swelling. Morphologies of hydrogels swollen at different $\mathrm{pH}$ values are shown in Figure 2 (although SEM is useful to reveal the hydrogel structure, care must be taken to avoid affecting the morphology during sample preparation [41]).

The hydrogel swollen at $\mathrm{pH}=2$ shows a compact and collapsed structure with few pores of about $10 \mu \mathrm{m}$ diameter (Figure 2A). The material swollen at $\mathrm{pH}=7$ (Figure 2B) shows a distribution of pores with an average diameter close to $10 \mu \mathrm{m}$. The hydrogel swollen at $\mathrm{pH}=13$ (Figure 2C) presents larger pores (diameters higher than $20 \mu \mathrm{m}$ ) and thinner walls, in agreement with its high swelling capacity. Based on these results it was considered of interest to compare the behavior of non-hydrolyzed hydrogels,

Figure 2. SEM micrographs of freeze-dried hydrogels after reaching equilibrium at: $\mathrm{A}$ ) $\mathrm{pH}=2, \mathrm{~B}$ ) $\mathrm{pH}=7$ and $\mathrm{C}) \mathrm{pH}=13$ 


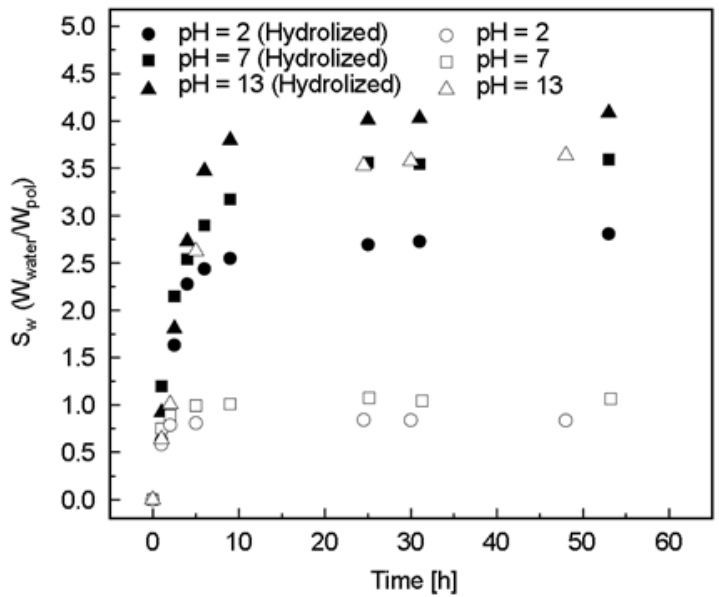

Figure 3. Swelling of hydrolyzed and non-hydrolyzed hydrogels at $\mathrm{pH}=2,7$ and 13

poly(HEMA-co-AAm), and hydrolyzed hydrogels, poly(HEMA-co-AA), obtained by immersing the non-hydrolyzed hydrogels in a buffer solution of $\mathrm{pH}=13$ for $48 \mathrm{~h}$, followed by a water extraction and drying procedure similar to the one used for the non-hydrolyzed hydrogels described in the experimental section.

A comparison of the swelling behavior of hydrolyzed and non-hydrolyzed hydrogels at different $\mathrm{pHs}$ is shown in Figure 3. In hydrolyzed hydrogels amide groups were converted to carboxylic acid groups $[19,42-45]$ whose degree of ionization depends on the $\mathrm{pH}$ of the swelling test. The particular curve obtained for the non-hydrolyzed gel at $\mathrm{pH}=13$ should not be considered for the discussion because partial conversion of amide to carboxylate groups takes place during the swelling test. Hydrolyzed hydrogels exhibited a larger swelling capacity than non-hydrolyzed hydrogels at any $\mathrm{pH}$. This can be ascribed to the higher hydrophilicity of carboxylic acid groups compared to amide groups and their partial ionization when increasing $\mathrm{pH}$.

\subsection{Release of dyes from non-hydrolyzed hydrogels}

After $24 \mathrm{~h}$ immersion in a water solution of a particular dye, the amount that was loaded to the hydrogel (defined as $M_{\infty}$ in mass per unit mass of polymer), was $M_{\infty}$ (Saf) $=42 \mathrm{mg} / \mathrm{g}$ and $M_{\infty}(\mathrm{Rf})=5 \mathrm{mg} / \mathrm{g}$. The significant loading observed for Saf evidences the presence of specific electrostatic interactions between the cation and functional groups of the hydrogel. This also causes a significant slower release rate of Saf with respect to $\mathrm{Rf}$ as is shown in

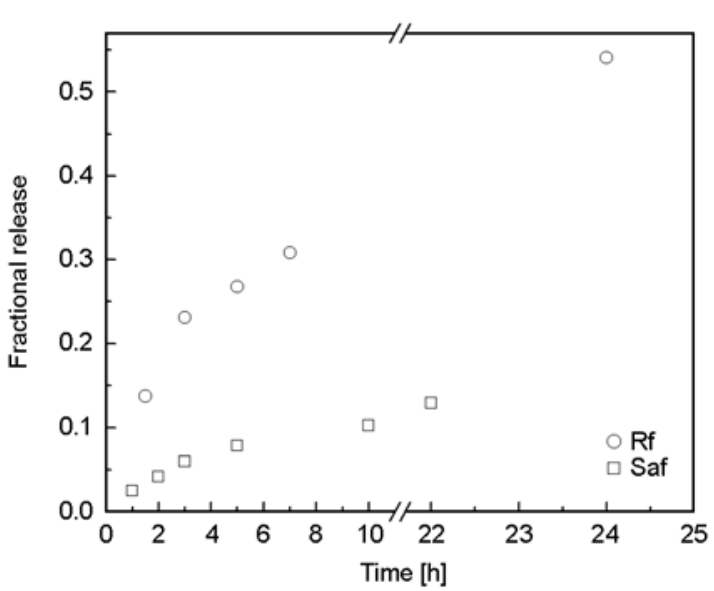

Figure 4. Fractional release of $\mathrm{Rf}$ and $\mathrm{Saf}$ at $\mathrm{pH}=7$ from non-hydrolyzed hydrogels

Figure 4 for $\mathrm{pH}=7$. The cumulative fractional release of Saf and Rf, $M_{\mathrm{t}} / M_{\infty}$, in a buffer medium at $\mathrm{pH}=7$ are shown in Figure 4. Therefore, the poly (HEMA-co-AAm) hydrogel might be suitable for the loading and slow release of cationic drugs.

An empiric equation developed by Peppas and coworkers for the release rate assumes a time-dependent power law function [46-47] presented in Equation (2):

$\frac{M_{\mathrm{t}}}{M_{\infty}}=k t^{\mathrm{n}}$

The fitting of experimental results with Equation (2) written in logarithmic form is shown in Figure 5. The resulting parameters are summarized in Table 1. When the value of the exponent is $n=0.5$, the release rate follows Fick's law. Within experimental error the release rate of the anionic dye follows a

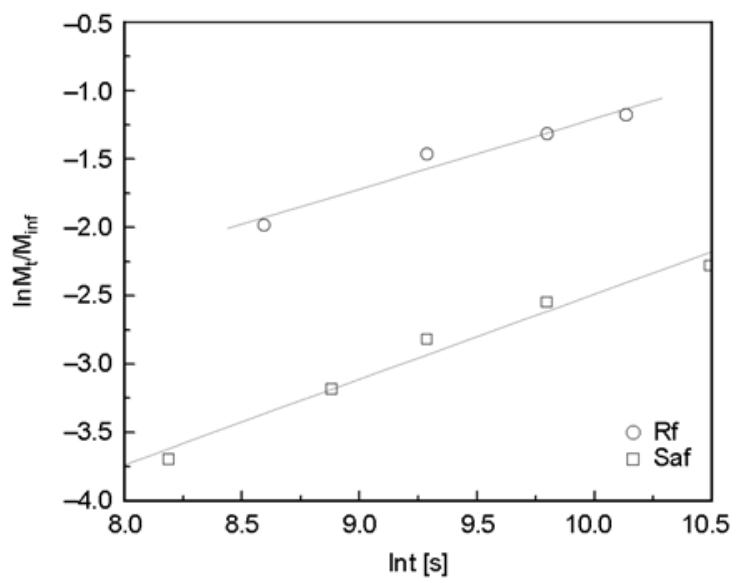

Figure 5. Logarithmic expression of the fractional release of $\mathrm{Rf}$ and $\mathrm{Saf}$ at $\mathrm{pH}=7$ from non-hydrolyzed hydrogels against natural logarithm of time expressed in seconds 
Table 1. Release characteristics of model drugs from poly(HEMA-co-AAm) hydrogels

\begin{tabular}{|c|c|c|c|c|}
\hline Drug & $\mathbf{k}\left[\mathbf{h}^{-\mathbf{n}}\right]$ & $\mathbf{n}$ & $\mathbf{R}$ & $\mathbf{D}\left[\mathbf{c m}^{\mathbf{2}} \cdot \mathbf{s}^{\mathbf{- 1}}\right]$ \\
\hline $\mathrm{Rf}$ & $1.74 \cdot 10^{-3}$ & 0.52 & 0.978 & $1.98 \cdot 10^{-8}$ \\
\hline Saf & $1.63 \cdot 10^{-4}$ & 0.62 & 0.987 & - \\
\hline
\end{tabular}

Fickian behavior while the cationic dye exhibits a non-Fickian mechanism. The departure from Fick's law can again be explained by the presence of electrostatic interactions between the positive charge of Saf and functional groups of the hydrogel. Similar results were observed for crystal violet when it was employed as model drug in poly(HEMA-co-AA) hydrogels [7]. The non-Fickian mechanism was ascribed to electrostatic interactions between the positive charge of the dye with carboxylic groups of the hydrogel [7].

For systems following a Fickian behavior, diffusion coefficients $(D)$ may be calculated from the slope of the plot of $M_{\mathrm{t}} / M_{\infty} v s . t^{1 / 2}$ (the initial slope is equal to $4 D^{1 / 2} /\left(\pi^{1 / 2} L\right)$, where $L$ is the slab thickness [25]). The diffusion coefficient of $\mathrm{Rf}$ reported in Table 1 lies in the same range as some of the values reported in the literature $[25,32]$.

\subsection{Release of Saf from a hydrolyzed hydrogel}

The possibility of varying the specific interactions of Saf by converting amide groups into carboxylic acid groups was investigated. It was found that the value of $M_{\infty}$ increased from $42 \mathrm{mg} / \mathrm{g}$ for the nonhydrolyzed hydrogel to $80 \mathrm{mg} / \mathrm{g}$ for the hydrolyzed hydrogel, implying that carboxylic acid groups promoted higher specific interactions with Saf than

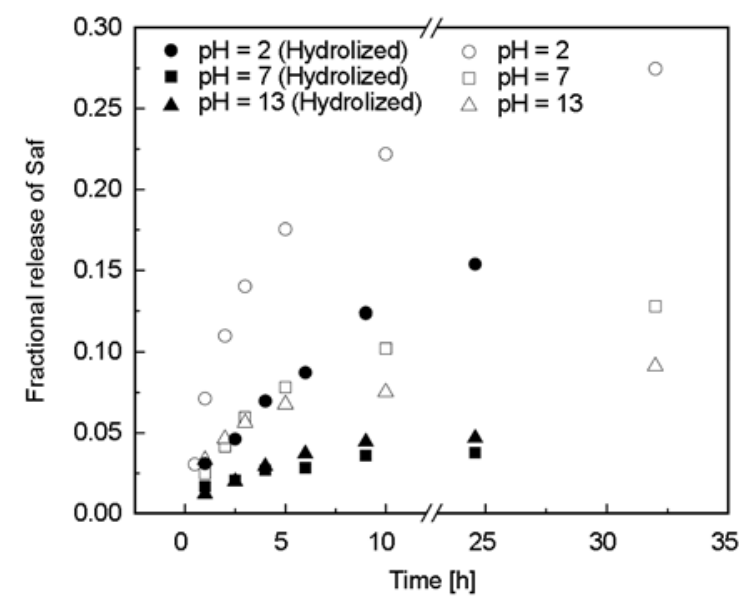

Figure 6. Fractional release of Saf for hydrolyzed and nonhydrolyzed hydrogels at $\mathrm{pH}=2,7$ and 13 amide groups. This was confirmed by comparing release rates of Saf from hydrolyzed and nonhydrolyzed hydrogels at $\mathrm{pH}$ 2, 7 and 13 (Figure 6). Again the partial conversion of amide to carboxylate groups, that takes place during the swelling test, must be considered for the curves at $\mathrm{pH}=13$.

For both types of hydrogels release rates were higher under acid conditions where the lowest swelling had been observed. This means that release rate depends more on specific interactions of the dye with the functional groups of the gel than on the swelling of the materials. Specific interactions between Saf and carboxylic acid groups should be stronger than the ones of Saf and acrylamide groups as evidenced by the significant decrease of the fractional release rate observed for hydrolyzed hydrogels at all $\mathrm{pH}$ values. This agrees with the higher loading capacity for Saf exhibited by hydrolyzed hydrogels. Specific interactions should decrease their strength under acid conditions to explain the highest release rate observed at $\mathrm{pH}=2$. This means that specific interactions of Saf should decrease in the sequence $\mathrm{COO}^{-}$ $>\mathrm{COOH}>\mathrm{CONH}_{2}$. Poly(HEMA-co-AA) hydrogels are therefore suitable for the very slow release of a cationic drug, particularly under neutral or alkaline $\mathrm{pH}$ values.

\section{Conclusions}

In conclusion, we showed that tuning the ionic character of a drug and the conversion of amide groups into carboxylate groups enables to control the loading capacity and the release rate from poly(HEMAco-AAm) hydrogels used in drug-delivery applications. These hydrogels are more affine with cationic than with anionic drugs. This leads to a higher loading capacity and a slower release rate of cationic dyes with respect to anionic dyes. Conversion of the hydrogel to poly(HEMA-co-AA) by hydrolysis at $\mathrm{pH}=13$, produced a significant increase in swelling and loading capacity and a decrease of the release rate, particularly under neutral or alkaline conditions. This means that specific interactions of the cationic dye decrease in the sequence $\mathrm{COO}^{-}>$ $\mathrm{COOH}>\mathrm{CONH}_{2}$.

\section{Acknowledgements}

The financial support of Universidad Nacional de Mar del Plata, Universidad Nacional de Río Cuarto, CONICET and ANPCyT (PICT 07-346), Argentina, is gratefully acknowledged. 


\section{References}

[1] Hoffman A. S.: Applications of thermally reversible polymers and hydrogels in therapeutics and diagnostics. Journal of Controlled Release, 6, 297-305 (1987). DOI: 10.1016/0168-3659(87)90083-6

[2] Peppas N. A., Hilt J. Z., Khademhosseini A., Langer R.: Hydrogels in biology and medicine: From molecular principles to bionanotechnology. Advanced Materials, 18, 1345-1360 (2006).

DOI: $10.1002 /$ adma.200501612

[3] Peppas N. A.: Hydrogels in medicine and pharmacy. CRC Press, Boca Raton (1987).

[4] Oh J. K., Drumright R., Siegwart D. J., Matyjaszewski K.: The development of microgels/nanogels for drug delivery applications. Progress in Polymer Science, 33, 448-477 (2008).

DOI: $10.1016 /$ j.progpolymsci.2008.01.002

[5] Tanaka T., Filmore D. J.: Kinetics of swelling of gels. Journal of Chemical Physics, 70, 1214-1218 (1979). DOI: $10.1063 / 1.437602$

[6] Tang Q., Wu J., Lin J., Li Q., Fan S.: Two-step synthesis of polyacrylamide/polyacrylate interpenetrating network hydrogels and its swelling/deswelling properties. Journal of Materials Science, 43, 5884-5890 (2008).

DOI: 10.1007/s10853-008-2857-x

[7] Yue Y., Sheng X., Wang P.: Fabrication and characterization of microstructured and $\mathrm{pH}$ sensitive interpenetrating networks hydrogel films and application in drug delivery field. European Polymer Journal, 45, 309-315 (2009).

DOI: $10.1016 /$ j.eurpolymj.2008.10.038

[8] Wichterle O., Lim D.: Hydrophilic gels for biological use. Nature, 185, 117-118 (1960).

DOI: $10.1038 / 185117 \mathrm{a} 0$

[9] Kabra B. G., Gehrke S. H., Hwang S. T., Ritschel W. A.: Modification of the dynamic swelling behavior of poly(2-hydroxyethyl methacrylate) in water. Journal of Applied Polymer Science, 42, 2409-2416 (1991). DOI: 10.1002/app.1991.070420906

[10] Wang J., Wu W.: Swelling behaviors, tensile properties and thermodynamic studies of water sorption of 2hydroxyethyl methacrylate/epoxy methacrylate copolymeric hydrogels. European Polymer Journal, 41, 1143-1151 (2005).

DOI: $10.1016 /$ j.eurpolymj.2004.11.034

[11] Huang C-W., Sun Y-M., Huang W-F.: Curing kinetics of the synthesis of poly(2-hydroxyethyl methacrylate) (PHEMA) with ethylene glycol dimethacrylate (EGDMA) as a crosslinking agent. Journal of Polymer Science Part A: Polymer Chemistry, 35, 1873-1889 (1997).

DOI: $10.1002 /($ SICI)1099-0518(19970730)35:10<1873 ::AID-POLA2>3.0.CO;2-P
[12] Podual K., Doyle III F. J., Peppas N. A.: Dynamic behavior of glucose oxidase-containing microparticles of poly(ethylene glycol)-grafted cationic hydrogels in an environment of changing $\mathrm{pH}$. Biomaterials, 21, 1439-1450 (2000).

DOI: $10.1016 / \mathrm{S} 0142-9612(00) 00020-\mathrm{X}$

[13] Anseth K. S., Scott R. A., Peppas N. A.: Effects of ionization on the reaction behavior and kinetics of acrylic acid polymerizations. Macromolecules, 29, 83088312 (1996).

DOI: $10.1021 / \mathrm{ma960840 \textrm {r }}$

[14] Li L., Lee L. J.: Photopolymerization of HEMA/ DEGDMA hydrogels in solution. Polymer, 46, 1154011547 (2005). DOI: $10.1016 /$ j.polymer.2005.10.051

[15] Lu S., Anseth K. S.: Photopolymerization of multilaminated poly(HEMA) hydrogels for controlled release. Journal of Controlled Release, 57, 291-300 (1999). DOI: $10.1016 / \mathrm{S} 0168-3659(98) 00125-4$

[16] Bryant S. J., Anseth K. S.: The effects of scaffold thickness on tissue engineered cartilage in photocrosslinked poly(ethylene oxide) hydrogels. Biomaterials, 22, 619-626 (2001).

DOI: $10.1016 / \mathrm{S} 0142-9612(00) 00225-8$

[17] Burdick J. A., Anseth K. S.: Photoencapsulation of osteoblasts in injectable RGD-modified PEG hydrogels for bone tissue engineering. Biomaterials, 23, 4315-4323 (2002).

DOI: $10.1016 / \mathrm{S} 0142-9612(02) 00176-\mathrm{X}$

[18] Nizam El-Din H. M., El-Naggar A. W. M.: Synthesis and characterization of hydroxyethyl methacrylate/ acrylamide responsive hydrogels. Journal of Applied Polymer Science, 95, 1105-1115 (2005).

DOI: $10.1002 /$ app. 21326

[19] Gómez M. L., Fasce D. P., Williams R. J. J., ErraBalsells R., Fatema M. K., Nonami H.: Silsesquioxane functionalized with methacrylate and amine groups as a crosslinker/co-initiator for the synthesis of hydrogels by visible-light photopolymerization. Polymer, 49, 3648-3653 (2008).

DOI: $10.1016 /$ j.polymer.2008.07.001

[20] Salomone J. C.: Polymeric materials encyclopedia, Vol 7. CRC press, Boca Raton (1996).

[21] Encinas M. V., Rufs A. M., Neumann M. G., Previtali C. M.: Photoinitiated vinyl polymerization by safranine $\mathrm{T} /$ triethanolamine in aqueous solution. Polymer, 37, 1395-1398 (1996). DOI: 10.1016/0032-3861(96)81137-2

[22] Encinas M. V., Previtali C. M., Gehlen M. H., Neumann M. G.: The interaction of aliphatic amines with safranine $\mathrm{T}$ in aqueous solution. Journal of Photochemistry and Photobiology A: Chemistry, 94, 237-241 (1996). DOI: $10.1016 / 1010-6030(95) 04218-0$ 
[23] Previtali C. M., Bertolotti S. G., Neumann M. G., Pastre I. A., Rufs A. M., Encinas M. V.: Laser flash photolysis study of the photoinitiator system safranine T-aliphatic amines for vinyl polymerization. Macromolecules, 27, 7454-7458 (1994).

DOI: $10.1021 / \mathrm{ma} 00103 \mathrm{a} 031$

[24] Gómez M. L., Avila V., Montejano H. A., Previtali C. M.: A mechanistic and laser flash photolysis investigation of acrylamide polymerization photoinitiated by the three component system safranine-T/triethanolamine/diphenyliodonium chloride. Polymer, 44, 28752881 (2003).

DOI: 10.1016/S0032-3861(03)00212-X

[25] Tomić S. L., Dimitrijević S. I., Marinković A. D., Najman S., Filipović J. M.: Synthesis and characterization of poly(2-hydroxyethyl methacrylate/itaconic acid) copolymeric hydrogels. Polymer Bulletin, 63, 837851 (2009).

DOI: 10.1007/s00289-009-0123-2

[26] Moogooee M., Ramezanzadeh H., Jasoori S., Omidi Y., Davaran S.: Synthesis and in vitro studies of crosslinked hydrogel nanoparticles containing amoxicillin. Journal of Pharmaceutical Sciences, 100, 1057-1066 (2011).

DOI: 10.1002/jps.22351

[27] Abd Alla S. G., Nizam El-Din H. M., El-Naggar A. W. M.: Structure and swelling-release behaviour of poly (vinyl pyrrolidone) (PVP) and acrylic acid (AAc) copolymer hydrogels prepared by gamma irradiation. European Polymer Journal, 43, 2987-2998 (2007). DOI: 10.1016/j.eurpolymj.2007.04.016

[28] Agarwal S., Sumana G., Gupta D. C.: Poly (2-hydroxyethyl methacrylate)-based hydrogels for slow release of pralidoxime chloride. Journal of Applied Polymer Science, 66, 267-270 (1997).

DOI: $10.1002 /($ SICI) 1097-4628(19971010)66:2<267:: AID-APP7>3.0.CO;2-U

[29] Sastre R. L., Blanco M. D., Gómez C., del Socorro J. M., Teijón J. M.: Cytarabine trapping in poly(2hydroxyethyl methacrylate-co-acrylamide) hydrogels: Drug delivery studies. Polymer International, 48, 843850 (1999).

DOI: $10.1002 /(\mathrm{SICI}) 1097-0126(199909) 48: 9<843:$ : AID-PI225>3.0.CO;2-8

[30] Suhag G. S., Bhatnagar A., Singh H.: Poly(hydroxyethyl methacrylate)-based co-polymeric hydrogels for transdermal delivery of salbutamol sulphate. Journal of Biomaterials Science, Polymer Edition, 19, 11891200 (2008).

DOI: $10.1163 / 156856208785540118$

[31] Xu J., Li X., Sun F.: Cyclodextrin-containing hydrogels for contact lenses as a platform for drug incorporation and release. Acta Biomaterialia, 6, 486-493 (2010).

DOI: $10.1016 /$ j.actbio.2009.07.021
[32] Kalagasidis Krušić M., Ilić M., Filipović J.: Swelling behavior and paracetamol release from $\operatorname{poly}(\mathrm{N}$-isopropylacrylamide-itaconic acid) hydrogels. Polymer Bulletin, 63, 197-211 (2009).

DOI: $10.1007 / \mathrm{s} 00289-009-0086-3$

[33] Krishna Rao K. S. V., Ha C. S.: pH sensitive hydrogels based on acryl amides and their swelling and diffusion characteristics with drug delivery behavior. Polymer Bulletin, 62, 167-181 (2009).

DOI: $10.1007 / \mathrm{s} 00289-008-0011-1$

[34] Harling S., Schwoerer A., Scheibe K., Daniels R., Menzel H.: A new hydrogel drug delivery system based on hydroxyethylstarch derivatives. Journal of Microencapsulation, 27, 400-408 (2010).

DOI: $10.3109 / 02652040903367301$

[35] García D. M., Escobar J. L., Noa Y., Bada N., Hernáez E., Katime I.: Timolol maleate release from pH-sensible poly(2-hydroxyethyl methacrylate-co-methacrylic acid) hydrogels. European Polymer Journal, 40, 16831690 (2004).

DOI: 10.1016/j.eurpolymj.2004.03.012

[36] García O., Blanco M. D., Martín J. A., Teijón J. M.: 5fluorouracil trapping in poly(2-hydroxyethyl methacrylate-co-acrylamide) hydrogels: In vitro drug delivery studies. European Polymer Journal, 36, 111-122 (2000).

DOI: $10.1016 / \mathrm{S} 0014-3057(99) 00037-3$

[37] Qiu Y., Park K.: Environment-sensitive hydrogels for drug delivery. Advanced Drug Delivery Reviews, 53, 321-339 (2001). DOI: $10.1016 / \mathrm{S} 0169-409 \mathrm{X}(01) 00203-4$

[38] Lin G., Chang S., Kuo C-H., Magda J., Solzbacher F.: Free swelling and confined smart hydrogels for applications in chemomechanical sensors for physiological monitoring. Sensors and Actuators B: Chemical, 136, 186-195 (2009). DOI: $10.1016 /$ j.snb.2008.11.001

[39] Liu F., Urban M. W.: Recent advances and challenges in designing stimuli-responsive polymers. Progress in Polymer Science, 35, 3-23 (2010). DOI: $10.1016 /$ j.progpolymsci.2009.10.002

[40] Wang J., Gao P., Ye L., Zhang A-Y., Feng Z-G.: Dual thermo-responsive polyrotaxane-based triblock copolymers synthesized via ATRP of $\mathrm{N}$-isopropylacrylamide initiated with self-assemblies of $\mathrm{Br}$ end-capped Pluronic F127 with $\beta$-cyclodextrins. Polymer Chemistry, 2, 931-940 (2011).

DOI: $10.1039 / \mathrm{COPY} 00360 \mathrm{C}$

[41] Hong P-D., Chen J-H.: Network structure and chain mobility of freeze-dried polyvinyl chloride/dioxane gels. Polymer, 39, 5809-5817 (1998). DOI: $10.1016 / \mathrm{S} 0032-3861(98) 00031-7$ 
[42] Lee W-F., Shieh C-H.: pH-thermoreversible hydrogels. I. Synthesis and swelling behaviors of the ( $N$-isopropylacrylamide-co-acrylamide- $c o$-2-hydroxyethyl methacrylate) copolymeric hydrogels. Journal of Applied Polymer Science, 71, 221-231 (1999).

DOI: 10.1002/(SICI)1097-4628(19990110)71:2<221::

$$
\text { AID-APP5>3.0.CO;2-W }
$$

[43] Kizilay M. Y., Okay O.: Effect of hydrolysis on spatial inhomogeneity in poly(acrylamide) gels of various crosslink densities. Polymer, 44, 5239-5250 (2003). DOI: 10.1016/S0032-3861(03)00494-4

[44] Pourjavadi A., Amini-Fazl M. S., Hosseinzadeh H.: Partially hydrolyzed crosslinked alginate-graft-polymethacrylamide as a novel biopolymer-based superabsorbent hydrogel having pH-responsive properties. Macromolecular Research, 13, 45-53 (2005).

DOI: $10.1007 / \mathrm{BF} 03219014$
[45] Marandi G. B., Esfandiari K., Biranvand F., Babapour M., Sadeh S., Mahdavinia G. R.: pH sensitivity and swelling behavior of partially hydrolyzed formaldehyde-crosslinked poly(acrylamide) superabsorbent hydrogels. Journal of Applied Polymer Science, 109, 1083-1092 (2008).

DOI: $10.1002 / a p p .28205$

[46] Peppas N. A., Bures P., Leobandung W., Ichikawa H.: Hydrogels in pharmaceutical formulations. European Journal of Pharmaceutics and Biopharmaceutics, 50, 27-46 (2000).

DOI: 10.1016/S0939-6411(00)00090-4

[47] Siepmann J., Peppas N. A.: Mathematical modeling of controlled drug delivery. Advanced Drug Delivery Reviews, 48, 137-138 (2001).

DOI: $10.1016 / \mathrm{S} 0169-409 \mathrm{X}(01) 00111-9$ 